\title{
Risk Factors and Outcomes of Opioid Users with and Without Concurrent Benzodiazepine Use in the North Carolina Medicaid Population
}

Anna Hung, PharmD, PhD, MS; Christopher Bush, MPH; Melissa Greiner, MS; Hilary Campbell, PharmD, JD; Bradley Hammill, DrPH; Matthew L. Maciejewski, PhD; and Aaron McKethan, PhD

\begin{abstract}
BACKGROUND: Concurrent use of opioids and benzodiazepines is associated with increased risk of opioid overdose and death. Clinical guidelines recommend against this practice and quality measures incentivize plans to minimize concurrent use.
\end{abstract}

OBJECTIVE: To compare comorbidities, risky opioid-related behaviors such as high daily doses or multiple prescribers or pharmacies, and outcomes of users of opioids with and without benzodiazepine in the 2017-2018 North Carolina Medicaid population.

METHODS: This was a retrospective claims analysis that used 2017-2018 North Carolina Medicaid enrollment and administrative claims data to describe 3 populations: (1) opioid users who concurrently used benzodiazepine for at least 30 days, (2) opioid users who used some benzodiazepine for 0 to less than 30 overlapping days, and (3) opioid users who did not use benzodiazepines.

RESULTS: From 2017 to 2018, 6\% of opioid users concurrently used opioids and benzodiazepines for at least 30 days, and $14 \%$ used some benzodiazepine for less than 30 overlapping days. Persons filling prescriptions for opioids and benzodiazepines were more likely to have mood disorders and more likely to have depression than opioid users who did not use benzodiazepines. Compared with those not using benzodiazepines, opioid users using benzodiazepine were also more likely to have higher daily opioid doses (at least 90 morphine milligram equivalents), at least 3 prescribers, and at least 3 pharmacies for opioid prescriptions. Although enrollees with at least 30 days of overlapping benzodiazepines and opioids had a higher percentage diagnosed with opioid use disorder compared with those with less than 30 days ( $30 \%$ vs. $13 \%$ ), a similar percentage received medication-assisted treatment continuously for 90 days ( $2.6 \%$ vs. $2.7 \%)$ during 2017-2018. Users of opioids and benzodiazepines, whether for at least 30 overlapping days or less, had higher 1-year cumulative incidences of allcause outpatient emergency department visits (64\% and $65 \%$ vs. $52 \%)$ and all-cause hospitalizations ( $25 \%$ and $21 \%$ vs. $14 \%$ ) compared with opioid users without benzodiazepine use.

CONCLUSIONS: Despite guidelines and quality measures, patients continue to use opioids and benzodiazepines concurrently. Addressing underlying mood disorders and depression, curbing risky opioid-related behaviors, and increasing access to medication-assisted treatment may benefit this population.

J Manag Care Spec Pharm. 2020;26(2):169-75

Copyright $\odot 2020$, Academy of Managed Care Pharmacy. All rights reserved.

\section{What is already known about this subject}

Concurrent use of opioids and benzodiazepines is associated with increased risk of opioid overdose and death.

In 2016, the Centers for Disease Control and Prevention (CDC) released guidelines advising clinicians to avoid prescribing opioids and benzodiazepines concurrently, and the U.S. Food and Drug Administration (FDA) added black box warnings to opioids and benzodiazepines regarding concurrent use.

In 2018, a quality measure focusing on concurrent use of opioids and benzodiazepines for at least 30 days was added to the Medicaid Adult Core Set.

\section{What this study adds}

During 2017-2018, 6\% of the North Carolina Medicaid population used opioids and benzodiazepines for at least 30 overlapping days, and 14\% used opioids and some benzodiazepine (0-29 overlapping days).

Although current quality measures focus on the concurrent use of opioids and benzodiazepines for at least 30 overlapping days, this study shows that those who use opioids and some benzodiazepine (0-29 overlapping days) also have high rates of emergency department visits and hospitalizations.

The differences in mental health comorbidities and risky opioidrelated behaviors between concurrent and nonconcurrent user populations described in this study can help inform the design of potential clinical and utilization management programs as the North Carolina Medicaid program undergoes a transformation from fee for service to managed care.

T he concurrent use of an opioid and a benzodiazepine increases risk of opioid overdose, emergency department visits, inpatient admissions, and death. ${ }^{1-6}$ This increase of risk is likely because of the increased risk of respiratory depression when opioids are used with benzodiazepines. ${ }^{7}$ In March 2016, the Centers for Disease Control and Prevention (CDC) advised clinicians to avoid prescribing opioids and benzodiazepines concurrently whenever possible as part of its "Guideline for Prescribing Opioids for Chronic Pain." "7,8 In August 2016, the U.S. Food and Drug Administration (FDA) added black box warnings to all drugs in the opioid and 


\section{Risk Factors and Outcomes of Opioid Users with and Without Concurrent Benzodiazepine Use in the North Carolina Medicaid Population}

benzodiazepine drug classes. In 2018, a measure developed by the Pharmacy Quality Alliance (PQA) depicting concurrent use of an opioid and a benzodiazepine for at least 30 days was added to the Medicaid Adult Core Set, which is a set of health care quality measures for adult Medicaid enrollees, as well as the Medicaid 1115 Substance Use Disorder Demonstrations.

Despite these policy and reporting recommendations, the concurrent use of opioids and benzodiazepines continues to occur. What remains unknown is how concurrent users differ from those using opioids alone in terms of demographics and comorbidities and whether they exhibit risky opioid-related behaviors, such as receipt of high doses of opioids and opioids from multiple prescribers and/or pharmacies. The results of this study will help providers and payers design programs and initiatives to address this risky population.

\section{Methods}

We used 2017-2018 North Carolina (NC) Medicaid enrollment and claims data to identify enrollees who filled at least 1 opioid prescription. Due to data access limitations, we excluded those enrollees aged over 65 years or who had Medicare dual eligibility. We required enrollment at the cohort index date and for each prescription that was analyzed. We identified all prescriptions for opioids and benzodiazepines using National Drug Code numbers linked to the CDC's morphine milligram equivalents (MME) table. ${ }^{9}$ We then subdivided this population into 3 mutually exclusive comparison groups: (1) those who continuously used an opioid and a benzodiazepine with at least 30 days of overlap (modeled after the PQA measure ${ }^{10}$ ); (2) those who used an opioid and some benzodiazepines (but with 0 to less than 30 days of overlap); and (3) those who used an opioid but did not use a benzodiazepine.

Baseline demographics, selected diagnosed comorbidities, prescription opioid-related characteristics, receipt of medication-assisted treatment, and 1-year outcomes were compared between the 3 groups. Demographics included age, sex, and race/ethnicity, along with Medicaid eligibility category, managed care region, and residence type. The 6 managed care regions represented the proposed regions in NC's Medicaid transformation. ${ }^{11}$ Selected diagnosed comorbidities included mood disorders, depression, schizophrenia, any cancer, and chronic pain and were identified based on at least 1 prespecified International Classification of Diseases, Tenth Revision, Clinical Modification (ICD-10-CM) code (see Appendix A and Appendix B, available in online article). Similar to past studies that evaluated opioid and benzodiazepine use, mental health comorbidities were reported. ${ }^{1,6,12}$ Cancer and chronic pain were selected because higher doses and longer durations of opioid use are often seen for these indications.

Prescription opioid-related characteristics per enrollee included receipt of at least 90 daily MME; receipt of at least a continuous 90-day supply of 90 daily MME; use of at least 3 prescribers; use of at least 3 pharmacies; and use of at least 4 prescribers and at least 4 pharmacies to fill opioid prescriptions at any point in 2017-2018. Comorbidities were identified using the 90 days before the index date for each group, with index dates defined as the 30th day of continuous opioid and benzodiazepine overlap for cohort 1 and first date of opioid prescription for cohorts 2 and 3.

Medication-assisted treatment included prescriptions for the tablet and film formulations of generic buprenorphine or buprenorphine and naloxone in combination. One-year outcomes included all-cause mortality, all-cause hospitalization, outpatient emergency department (ED) visit, and diagnosis of an unintentional opioid overdose. These outcomes were derived from at least 1 prespecified Healthcare Common Procedure Coding System and/or ICD-10-CM code (see Appendix A).

\section{Statistical Analysis}

Baseline demographic and prescription opioid characteristics were described using frequencies with percentages for categorical variables and means with standard deviations for continuous variables. Chi-square and Kruskal-Wallis tests were used to test for differences across groups for categorical and continuous variables, respectively.

One-year cumulative incidence of all-cause mortality was calculated via the Kaplan-Meier function. One-year cumulative incidence of all-cause hospitalization, outpatient ED visit, and unintentional opioid overdose were calculated using the cumulative incidence function, accounting for the competing risk of death. ${ }^{13}$ For all-cause mortality, enrollees were censored at the earliest of the end of their Medicaid enrollment, the end of the period, or 1 year from their cohort index date. All other outcomes were additionally censored for mortality.

We tested for group differences in mortality using log-rank tests and for all other outcomes using Gray tests. ${ }^{14} \mathrm{~A} P$ value of less than 0.05 was considered statistically significant for all tests. All analyses were performed at the patient level and were done using SAS version 9.4 (SAS Institute, Cary, NC). This study was approved by the Duke University Health System Institutional Review Board.

\section{Results}

From 2017 to 2018, there were 2,823,633 enrollees in NC Medicaid. As shown in Figure 1, there were 2,457,174 persons aged under 65 years in NC who were enrolled in Medicaid and who were not dual eligible for Medicare. Of these, 322,835 (13\%) filled at least 1 opioid prescription. Of these opioid users, 18,197 (6\%) had concurrent opioid and benzodiazepine prescriptions that overlapped for at least 30 days; 45,242 (14\%) had prescriptions for an opioid and a benzodiazepine with 0-29 days of overlap; and the majority (80\%) did not have benzodiazepine use (Table 1). 


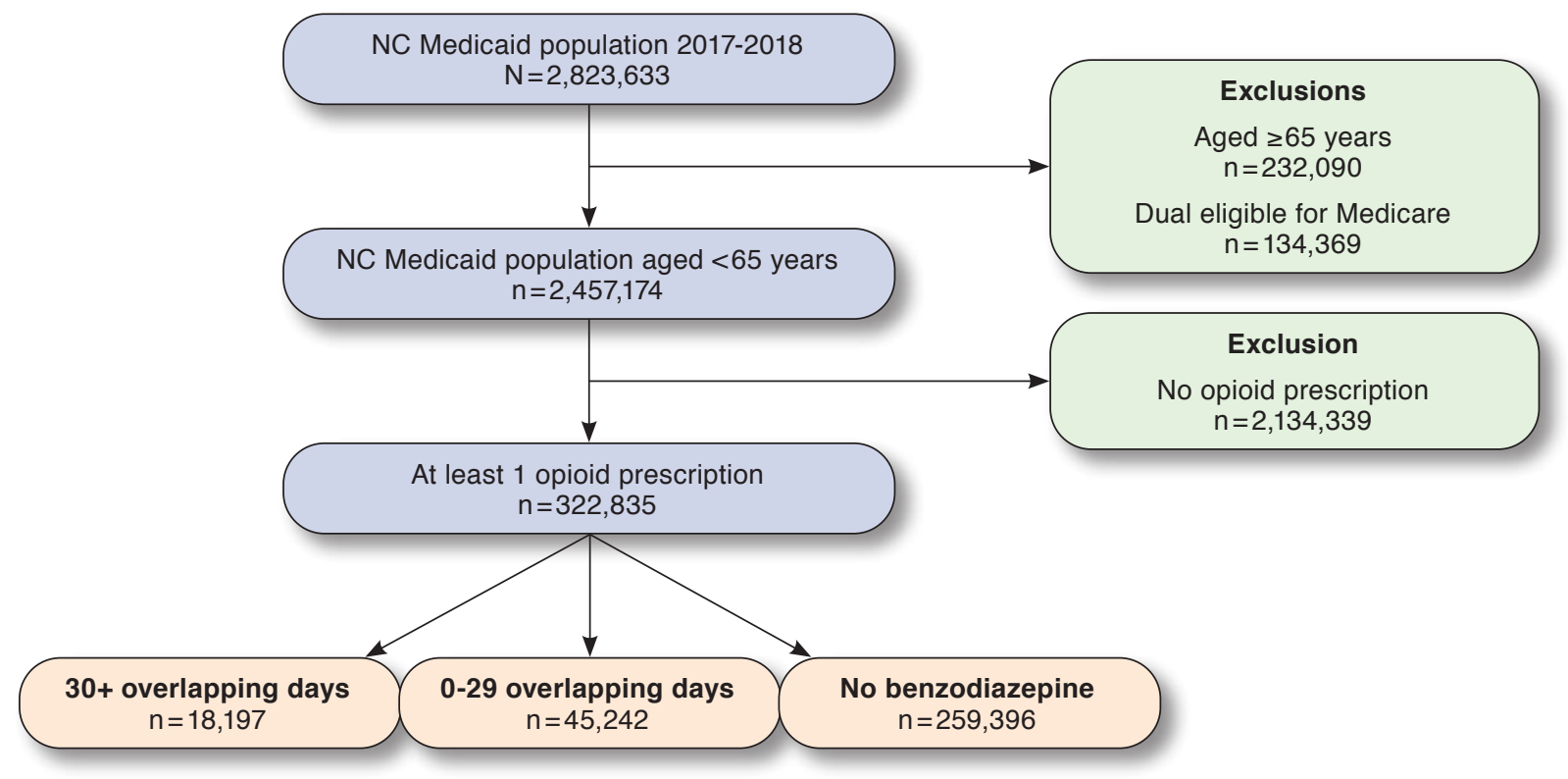

NC $=$ North Carolin .

\section{Differences in Patient Characteristics and Comorbidities}

Compared with opioid users with no benzodiazepine use, opioid users with at least 30 days and opioid users with less than 30 days of overlapping benzodiazepines were older (mean age of 47 and 36 years vs. 30 years), more likely to be white ( $75 \%$ and $67 \%$ vs. $48 \%$ ), and more likely to qualify for Medicaid based on disability (63\% and 36\% vs. 23\%). Those with concurrent use for at least 30 days and those with at least some benzodiazepine use also had higher percentages with diagnosed mental health-related comorbidities compared with opioid users with no benzodiazepine use (mood disorder: $41 \%$ and $29 \%$ vs. $11 \%$; depression: $40 \%$ and $28 \%$ vs. $12 \%$; schizophrenia: $5 \%$ and $4 \%$ vs. $1 \%$, respectively). All differences were statistically significant.

\section{Differences in Opioid Prescription Characteristics and Medication-Assisted Treatment}

Opioid users with at least 30 days of overlapping benzodiazepines and opioid users with some benzodiazepine use were more likely to fill a prescription with at least 90 daily MME in $2017-2018$ (32\% and 15\% vs. 6\%) compared with those with no benzodiazepine use. Moreover, opioid users with at least 30 days of overlapping benzodiazepines and opioid users with some benzodiazepine use were more likely to have filled opioid prescriptions from at least 3 prescribers or at least 3 pharmacies compared with opioid users with no benzodiazepine use (at least 3 prescribers: $49 \%$ and 24\% vs. 12\%, respectively; at least 3 pharmacies: $24 \%$ and $9 \%$ vs. $4 \%$, respectively).
Although enrollees with at least 30 days of overlapping benzodiazepines and opioids had a higher percentage diagnosed with opioid use disorder compared with those with less than 30 days overlap (30\% vs. 13\%; Table 2), similar percentages received medication-assisted treatment at all (4.4\% versus $4 \%)$ or continuously for 90 days (2.6\% versus $2.7 \%$ ) at any point in 2017-2018. Meanwhile, 9\% of opioid users with no benzodiazepine use were diagnosed with opioid use disorder, and $2.1 \%$ and $1.4 \%$ received medication-assisted treatment at all or continuously for 90 days, respectively.

\section{Differences in Outcomes}

Opioid users with at least 30 days of overlapping benzodiazepines and opioid users with some benzodiazepine use had higher 1-year cumulative incidences of outpatient ED visits (64\% and $65 \%$ vs. $52 \%$; Table 2 ) and hospitalizations (25\% and $21 \%$ vs. $14 \%$ ) compared with opioid users without benzodiazepine use. One-year cumulative incidences of all-cause mortality ( $4 \%$ and $2 \%$ vs. $1 \%$, respectively) and unintentional opioid overdose ( $1.1 \%$ and $0.4 \%$ vs. $0.2 \%$, respectively) were higher for opioid users with at least 30 days of overlapping benzodiazepines and opioid users with some benzodiazepine use versus opioid users with no benzodiazepine use.

\section{Discussion}

In a cohort of NC Medicaid enrollees in 2017-2018, we found that $6 \%$ of opioid users concurrently used a benzodiazepine 


\section{Risk Factors and Outcomes of Opioid Users with and Without Concurrent Benzodiazepine Use in the North Carolina Medicaid Population}

\section{TABLE 1 Demographics and Comorbidities of NC Medicaid Opioid Users with and Without Benzodiazepine Use}

\begin{tabular}{|c|c|c|c|c|c|}
\hline & $\begin{array}{c}\text { Total } \\
\text { Any Opioid Use }\end{array}$ & $\begin{array}{l}\text { Subgroup 1 } \\
\text { Overlapping } \\
\text { Benzodiazepine and } \\
\text { Opioid Use for } \\
\geq 30 \text { Days }\end{array}$ & $\begin{array}{c}\text { Subgroup } 2 \\
\text { Opioid and Some } \\
\text { Benzodiazepine Use }\end{array}$ & $\begin{array}{c}\text { Subgroup } 3 \\
\text { Opioid Use But No } \\
\text { Benzodiazepine Use }\end{array}$ & $P$ Value $^{\mathrm{a}}$ \\
\hline Number & 322,835 & 18,197 & 45,242 & 259,396 & \\
\hline \multicolumn{6}{|l|}{ Demographics } \\
\hline Age, mean (SD) & $31.6 \quad(15.6)$ & $47.2 \quad(10.6)$ & $36.2 \quad(14.7)$ & $29.7 \quad(15.3)$ & $<0.001$ \\
\hline Male sex, n (\%) & $100,832 \quad(31.2)$ & $5,267 \quad(28.9)$ & $11,493 \quad(25.4)$ & $84,072 \quad(32.4)$ & $<0.001$ \\
\hline \multicolumn{6}{|l|}{ Race, n (\%) } \\
\hline African American & $115,821 \quad(35.9)$ & $2,906 \quad(16.0)$ & $10,622 \quad(23.5)$ & $102,293 \quad(39.4)$ & $<0.001$ \\
\hline White & $168,011 \quad(52.0)$ & $13,592 \quad(74.7)$ & $30,182 \quad(66.7)$ & $124,237 \quad(47.9)$ & $<0.001$ \\
\hline Other race & $39,003 \quad(12.1)$ & $1,699 \quad(9.3)$ & 4,438 & $32,866 \quad(12.7)$ & $<0.001$ \\
\hline \multicolumn{6}{|l|}{ Medicaid eligibility $\mathrm{n}(\%)$} \\
\hline Average days of enrollment, mean (SD) & $428.0(225.6)$ & $532.1 \quad(196.5)$ & $461.1 \quad(215.3)$ & $408.6(223.7)$ & $<0.001$ \\
\hline Aid to families & $172,744 \quad(53.5)$ & $6,254 \quad(34.4)$ & $24,126 \quad(53.3)$ & $142,275 \quad(54.8)$ & $<0.001$ \\
\hline Aid to children & $41,425 \quad(12.8)$ & $20 \quad(0.1)$ & 3,240 & $38,165 \quad(14.7)$ & $<0.001$ \\
\hline Aid to disabled & $86,429 \quad(26.8)$ & $11,431 \quad(62.8)$ & $16,280 \quad(36.0)$ & $58,802 \quad(22.7)$ & $<0.001$ \\
\hline Medicaid for pregnant women & $17,301 \quad(5.4)$ & $15 \quad(0.1)$ & $665 \quad(1.5)$ & $16,610 \quad(6.4)$ & $<0.001$ \\
\hline Refugee medical assistance & $(0.0)$ & - & - & $(0.1)$ & $<0.001$ \\
\hline Other & 4,778 & $(2.6)$ & $(2.1)$ & 3,389 & $<0.001$ \\
\hline \multicolumn{6}{|l|}{ Managed care region, $\mathrm{n}(\%)$} \\
\hline Region 1 (West, Asheville) & $33,787 \quad(10.5)$ & $2,594 \quad(14.3)$ & $7,566 \quad(16.7)$ & 23,624 & $<0.001$ \\
\hline Region 2 (Northwest) & $54,814 \quad(17.0)$ & $3,751 \quad(20.6)$ & $6,819 \quad(15.1)$ & $44,240 \quad(17.1)$ & $<0.001$ \\
\hline Region 3 (Southwest, Mecklenburg) & $72,436 \quad(22.4)$ & $3,843 \quad(21.1)$ & $11,276 \quad(24.9)$ & $57,324 \quad(22.1)$ & $<0.001$ \\
\hline Region 4 (North, Durham/Wake) & $53,548 \quad(16.6)$ & $2,032 \quad(11.2)$ & $6,507 \quad(14.4)$ & $45,004 \quad(17.3)$ & $<0.001$ \\
\hline Region 5 (South) & $63,901 \quad(19.8)$ & $3,631 \quad(20.0)$ & $7,125 \quad(15.7)$ & $53,152 \quad(20.5)$ & $<0.001$ \\
\hline Region 6 (East, Coastal) & $44,246 \quad(13.7)$ & $2,343 \quad(12.9)$ & $5,934 \quad(13.1)$ & $35,969 \quad(13.9)$ & $<0.001$ \\
\hline \multicolumn{6}{|l|}{ Residence, $\mathrm{n}(\%)$} \\
\hline Private living arrangement & $316,644 \quad(98.1)$ & $17,445 \quad(95.9)$ & $43,879 \quad(97.0)$ & $255,313 \quad(98.4)$ & $<0.001$ \\
\hline In SNF or domiciliary care & $2,088 \quad(0.6)$ & $416 \quad(2.3)$ & $543 \quad(1.2)$ & $1,134 \quad(0.4)$ & $<0.001$ \\
\hline In foster care or adoption home & 2,204 & - & $(0.5)$ & 1,985 & $<0.001$ \\
\hline Other living arrangement & 1,899 & $(1.8)$ & $(1.3)$ & $(0.4)$ & $<0.001$ \\
\hline \multicolumn{6}{|l|}{ Medical history, n (\%) } \\
\hline Mood disorders & $48,586 \quad(15.0)$ & $7,473 \quad(41.1)$ & $12,954 \quad(28.6)$ & $28,747 \quad(11.1)$ & $<0.001$ \\
\hline Depression & $49,500 \quad(15.3)$ & $7,209 \quad(39.6)$ & $12,850 \quad(28.4)$ & $30,077 \quad(11.6)$ & $<0.001$ \\
\hline Schizophrenia & $6,375 \quad(2.0)$ & $(4.8)$ & 1,865 & 3,671 & $<0.001$ \\
\hline Any cancer & $9,026 \quad(2.8)$ & $1,337 \quad(7.3)$ & 2,061 & 5,763 & $<0.001$ \\
\hline Chronic pain & $121,931 \quad(37.8)$ & $15,265 \quad(83.9)$ & $21,472 \quad(47.5)$ & $85,864 \quad(33.1)$ & $<0.001$ \\
\hline
\end{tabular}

Note: Cells with dashes indicate that small cell sizes $(<11)$ have been suppressed.

aHypothesis testing excluded the "any opioid Rx" group.

$N C=$ North Carolina; $R x=$ prescription; $S D=$ standard deviation; $S N F=$ skilled nursing facility.

for at least 30 days, and an additional 14\% had some use of benzodiazepine for 0-29 days of overlap. A past study found that in 2015, 28\% of opioid users in the Pennsylvania Medicaid population concurrently used benzodiazepines for at least 30 days. ${ }^{12}$ However, study authors excluded those aged $<18$ or $>64$ years, those with cancer, and those dual eligible for Medicare and Medicaid, and focused on patients more likely to have chronic as opposed to acute pain, per the PQA quality measure. ${ }^{12}$ In comparison, our study included patients aged $<65$ years and those with cancer. Another study in the Georgia Medicaid population found that in 2014, 10\%-15\% of opioid users had concurrent opioid and benzodiazepine use for at least 7 days. ${ }^{15}$ Across a commercial population, approximately 17\% of opioid users in 2013 also concurrently used benzodiazepines for at least 1 day. ${ }^{6}$ To our knowledge, our study is the first to describe the population that concurrently used opioids and benzodiazepines in a Medicaid population after the CDC and FDA recommendations in 2016.

Despite the lower percentage of concurrent use of opioids and benzodiazepines for at least 30 days, this continues to be an important population to monitor because of the multiple comorbidities and risky opioid-related behaviors, such as high 


\section{Risk Factors and Outcomes of Opioid Users with and Without Concurrent Benzodiazepine Use in the North Carolina Medicaid Population}

TABLE 2 Opioid Prescription Characteristics, Medication-Assisted Treatment, and Outcomes of NC Medicaid Opioid Users with and Without Benzodiazepine Use

\begin{tabular}{|c|c|c|c|c|c|}
\hline & $\begin{array}{c}\text { Total } \\
\text { Any Opioid Use }\end{array}$ & $\begin{array}{l}\text { Subgroup 1 } \\
\text { Overlapping } \\
\text { Benzodiazepine and } \\
\text { Opioid Use for } \\
\geq 30 \text { Days }\end{array}$ & $\begin{array}{c}\text { Subgroup 2 } \\
\text { Opioid and Some } \\
\text { Benzodiazepine Use }\end{array}$ & $\begin{array}{c}\text { Subgroup } 3 \\
\text { Opioid use But No } \\
\text { Benzodiazepine Use }\end{array}$ & $P$ Value ${ }^{a}$ \\
\hline \multicolumn{6}{|l|}{ Opioid prescription characteristics, $\mathbf{n}(\%)$} \\
\hline At least $90 \mathrm{MME}$ & $27,820 \quad(8.6)$ & $5,848(32.1)$ & $6,575(14.5)$ & $15,397 \quad(5.9)$ & $<0.001$ \\
\hline At least 90 days supply of $90 \mathrm{MME}$ & $10,211 \quad(3.2)$ & $4,492(24.7)$ & $1,010 \quad(2.2)$ & $4,709 \quad(1.8)$ & $<0.001$ \\
\hline At least 3 prescribers & $51,189 \quad(15.9)$ & $8,981 \quad(49.4)$ & $11,025 \quad(24.4)$ & $31,183(12.0)$ & $<0.001$ \\
\hline At least 3 pharmacies & $19,536 \quad(6.1)$ & $4,445 \quad(24.4)$ & $3,995 \quad(8.8)$ & $11,096 \quad(4.3)$ & $<0.001$ \\
\hline At least 4 prescribers +4 pharmacies & $3,782 \quad(1.2)$ & $846 \quad(4.6)$ & $918 \quad(2.0)$ & $2,018 \quad(0.8)$ & $<0.001$ \\
\hline \multicolumn{6}{|c|}{ Opioid or substance use disorder and MAT, n (\%) } \\
\hline Diagnosed with OUD & $28,921 \quad(9.0)$ & $5,446(29.9)$ & $6,004(13.3)$ & $17,471 \quad(6.7)$ & $<0.001$ \\
\hline Diagnosed with SUD & $48,424(15.0)$ & $7,621 \quad(41.9)$ & $9,770 \quad(21.6)$ & $31,033(12.0)$ & $<0.001$ \\
\hline Received MAT & $6,892 \quad(2.1)$ & $803 \quad(4.4)$ & $1,814 \quad(4.0)$ & $4,275 \quad(1.6)$ & $<0.001$ \\
\hline Continuously received 90 days of MAT & $4,491 \quad(1.4)$ & $470 \quad(2.6)$ & $1,232 \quad(2.7)$ & $2,789 \quad(1.1)$ & $<0.001$ \\
\hline \multicolumn{6}{|c|}{ Cumulative incidence of outcomes, \% (95\% CI) } \\
\hline All-cause mortality & $1.4 \quad(1.4-1.5)$ & $4.3 \quad(4.0-4.6)$ & $2.2 \quad(2.1-2.4)$ & $(1.1-1.2)$ & $<0.001$ \\
\hline All-cause hospitalization & $15.6(15.5-15.7)$ & $25.1 \quad(24.4-25.7)$ & $21.4 \quad(21.0-21.9)$ & $13.6(13.5-13.8)$ & $<0.001$ \\
\hline Outpatient ED visit & $54.4(54.2-54.6)$ & $63.9(63.1-64.6)$ & $64.7 \quad(64.2-65.1)$ & $51.5 \quad(51.3-51.8)$ & $<0.001$ \\
\hline Unintentional opioid overdose & $0.3 \quad(0.2-0.3)$ & $1.1 \quad(1.0-1.3)$ & $0.4 \quad(0.3-0.4)$ & $0.2 \quad(0.1-0.2)$ & $<0.001$ \\
\hline \multicolumn{6}{|c|}{$\begin{array}{l}\text { Note: All analyses are at the patient level. } \\
\text { aHypothesis testing excluded the "any opioid Rx" group. } \\
C I=\text { confidence interval; } E D=\text { emergency department; MAT=medication-assisted treatment; MME= morphine milligram equivalents; OUD=opioid use disorder; } \\
R x=\text { prescription; } S U D=\text { substance use disorder. }\end{array}$} \\
\hline
\end{tabular}

doses and multiple prescribers and pharmacies. In this study, approximately $40 \%$ of those who concurrently used an opioid and a benzodiazepine for at least 30 days had mood disorders compared with only $11 \%$ of opioid users who did not use benzodiazepines. Similar percentages were seen for depression. These results are similar to findings from other state Medicaid programs (60\% of the concurrent user population had mood disorders) and greater than what has been reported in commercial populations ( $17 \%$ of the concurrent user population had depression). ${ }^{6,12}$

Previous Medicaid studies have found that the concurrent use of opioids and benzodiazepines occurs most frequently out of all the potential opioid misuse measures, such as high doses, multiple prescribers, overlapping opioid prescriptions, and long-acting opioids for acute pain. ${ }^{12,15}$ Given that benzodiazepines are approved by the FDA for anxiety disorders, among several indications, and that Medicaid is the single largest payer for mental health services in the United States, ${ }^{16}$ the concurrent use of opioids and benzodiazepines will require special attention in Medicaid populations, unless the underlying mental health comorbidities in the population are reduced through appropriate treatment. Starting October 2019, the Substance Use-Disorder Prevention that Promotes Opioid Recovery and Treatment for Patients and Communities Act will require state Medicaid programs to monitor the concurrent use of opioids and benzodiazepines. Future research should evaluate whether this monitoring requirement reduces concurrent use in Medicaid populations.

In addition, the concurrent user group tended to have higher rates of risky opioid-related behaviors, such as prescriptions for high opioid doses and multiple prescribers and pharmacies, both of which are considered inappropriate and monitored by quality measures. ${ }^{10}$ Nearly one third of those who concurrently used an opioid and a benzodiazepine for at least 30 days also had at least 1 prescription with an average daily MME of at least 90 . Also, approximately one quarter had at least a continuous 90-day supply of 90 daily MME. Moreover, almost one half of concurrent users filled opioids from at least 3 different prescribers, and almost one quarter of concurrent users filled opioids from at least 3 pharmacies. Although the current PQA measure for multiple prescribers and pharmacies requires at least 4 prescribers and 4 pharmacies, evidence suggests that the use of at least 3 prescribers or at least 3 pharmacies is associated with increased opioid overdose and mortality. ${ }^{17}$ Designing utilization management programs to restrict patients from using high doses of opioids and multiple sources of prescribers or pharmacies for opioid prescriptions may be needed, especially in this population.

Although concurrent users of opioids and benzodiazepines for at least 30 days had more than twice the rate of opioid use 
disorder diagnosis compared with concurrent users of opioid and benzodiazepine for less than 30 days, rates of medicationassisted treatment were similar. This could indicate a gap in the use of medication-assisted treatment for health plans to address, especially as Medicaid increasingly plays a larger role in the reimbursement of substance use disorder services. ${ }^{16}$

Currently, the PQA quality measure in use for Medicaid populations (and endorsed by the National Quality Forum) focuses on the concurrent use of opioids and benzodiazepines for at least 30 days. However, this study found that concurrent users of opioids and benzodiazepines for at least 30 days, as well as opioid users who used some but less than 30 days of overlapping benzodiazepines, had high levels of ED visits and hospitalizations. A past study suggested that the elimination of the concurrent use of even 1 day of overlapping opioids and benzodiazepines could reduce the risk of ED visits and hospitalizations for opioid overdose by an estimated 15\%. ${ }^{6}$ Considering the high costs associated with ED visits and hospitalizations, it would be important for a health plan to monitor concurrent users of an opioid and a benzodiazepine for at least 30 days of overlap, as well as any overlap.

As NC Medicaid shifts from fee for service to managed care, health plans have an opportunity to decrease inappropriate opioid use, benzodiazepine use, and concurrent opioid and benzodiazepine use. A past study suggested that compared with fee for service, managed care Medicaid could be more effective at reducing inappropriate opioid prescribing. ${ }^{15}$ However, this is 1 study, and future research should evaluate whether pharmacy program management in fee-for-service versus managed care environments perform better at reducing inappropriate opioid use. At the least, health plans being held accountable to quality measures will want to understand their concurrent opioid and benzodiazepine user populations.

\section{Limitations}

This study examined Medicaid enrollees in NC, but findings may not apply beyond this population. Nevertheless, NC Medicaid's current transformation from fee for service to managed care makes this study population timely and relevant to these managed care plans. In addition, we did not require a full year of Medicaid eligibility to be included in the study; therefore, we may be missing enrollees who had overlapping benzodiazepine and opioid prescriptions for at least 30 days outside of their eligibility period. However, the average number of days of Medicaid enrollment was greater than 365.

Since this study used only enrollment and claims data, actual medication consumption may differ from the prescriptions filled. For example, medications paid for by cash were not captured in the claims data. Also, since unintentional opioid overdoses were measured based on ICD-10-CM codes, overdoses could be underestimated if some overdoses were rescued before medical care was sought. Finally, this is a descriptive study and does not evaluate causality.

\section{Conclusions}

Concurrent users of opioids and benzodiazepines can have more mental health comorbidities; higher prescription opioid doses; multiple prescription opioid prescribers and pharmacies; disproportionately lower medication-assisted treatment; and higher cumulative incidences of opioid overdose, ED visits, hospitalizations, and death than those using opioids alone. Providers and payers should address underlying mental health issues, curb risky opioid-related behaviors, and increase access to medication-assisted treatment.

\section{Authors}

ANNA HUNG, PharmD, PhD, MS, Duke Clinical Research Institute, Duke University, Durham, North Carolina. CHRISTOPHER BUSH, MPH; MELISSA GREINER, MS; HILARY CAMPBELL, PharmD, JD; BRADLEY HAMMILL, DrPH; and AARON MCKETHAN, PhD, Department of Population Health Sciences, Duke University, Durham, North Carolina. MATTHEW L. MACIEJEWSKI, PhD, Department of Population Health Sciences and Division of General Internal Medicine, Duke University, and Durham Center of Innovation to Accelerate Discovery and Practice Transformation, Durham Veterans Affairs Health Care System, Durham, North Carolina.

AUTHOR CORRESPONDENCE: Anna Hung, PharmD, PhD, MS, Duke Clinical Research Institute, Duke University, 200 Morris St., Durham, NC 27701. Tel.: 919.668.1493; E-mail: Anna.hung@duke.edu.

\section{DISCLOSURES}

This project was supported by Arnold Ventures (formerly Arnold Foundation). Hung reports personal fees from CVS Health and Blue Cross Blue Shield Association, unrelated to this work. Maciejewski reports Amgen stock ownership due to spouse employment, unrelated to this work. McKethan reports personal fees from North Carolina Department of Health and Human Services. All other authors have nothing to disclose.

Part of this content was presented as a poster at AMCP Nexus 2019; October 29-November 1, 2019; National Harbor, MD.

\section{REFERENCES}

1. Olfson M, Wall M, Wang S, Crystal S, Blanco C. Risks of fatal opioid overdose during the first year following nonfatal overdose. Drug Alcohol Depend. 2018;190:112-19.

2. Garg RK, Fulton-Kehoe D, Franklin GM. Patterns of opioid use and risk of opioid overdose death among Medicaid patients. Med Care. 2017;55(7):661-68.

3. Jones CM, McAninch JK. Emergency department visits and overdose deaths from combined use of opioids and benzodiazepines. Am J Prev Med. 2015;49(4):493-501

4. Dasgupta N, Funk MJ, Proescholdbell S, Hirsch A, Ribisl KM, Marshall S. Cohort study of the impact of high-dose opioid analgesics on overdose mortality. Pain Med. 2016;17(1):85-98. 


\section{Risk Factors and Outcomes of Opioid Users with and Without Concurrent Benzodiazepine Use in the North Carolina Medicaid Population}

5. Gomes T, Mamdani MM, Dhalla IA, Paterson JM, Juurlink DN. Opioid dose and drug-related mortality in patients with nonmalignant pain. Arch Intern Med. 2011;171(7):686-91.

6. Sun EC, Dixit A, Humphreys K, Darnall BD, Baker LC, Mackey S. Association between concurrent use of prescription opioids and benzodiazepines and overdose: retrospective analysis. BMJ. 2017;356:j760.

7. Dowell D, Haegerich TM, Chou R. CDC guideline for prescribing opioids for chronic pain-United States, 2016. JAMA. 2016;315(15):1624-45.

8. Dowell D, Haegerich TM, Chou R. CDC guideline for prescribing opioids for chronic pain-United States, 2016. MMWR Recomm Rep. 2016;65(1):1-49.

9. Centers for Disease Control and Prevention. Data resources. 2019. Available at: https://www.cdc.gov/drugoverdose/resources/data.html. Accessed January 12, 2020.

10. Pharmacy Quality Alliance. PQA core measure set. 2018. Available at: https://www.pqaalliance.org/opioid-core-measure-set. Accessed January 12, 2020.

11. North Carolina Department of Health and Human Services. About the NC Medicaid reform plan. March 30, 2016. Available at: https://www. ncdhhs.gov/nc-medicaid-reform/about-nc-medicaid-reform-plan. Accessed January 12, 2020.
12. Cochran G, Lo-Ciganic WH, Gellad WF, et al. Prescription opioid quality measures applied among Pennsylvania Medicaid enrollees. J Manag Care Spec Pharm. 2018;24(9):875-85. Available at: https://www.jmcp.org/ doi/10.18553/jmcp.2018.24.9.875.

13. Gray RJ. A class of K-sample tests for comparing the cumulative incidence of a competing risk. Ann Stat. 1988;16(3):1141-54. Available at: http:// www2.math.uu.se/ garmo/Grey.pdf. Accessed January 12, 2020.

14. Kalbfleisch JD, Prentice RL. The Statistical Analysis of Failure Time Data. New York: John Wiley \& Sons; 1980.

15. Jayawardhana J, Abraham AJ, Perri M. Opioid analgesics in Georgia Medicaid: trends in potential inappropriate prescribing practices by demographic characteristics, 2009-2014. J Manag Care Spec Pharm. 2018;24(9):886-94. Available at: https://www.jmcp.org/doi/10.18553/ jmcp.2018.24.9.886.

16. Medicaid.gov. Behavioral health services. Available at: https://www.medicaid.gov/medicaid/benefits/bhs/index.html. Accessed January 12, 2020.

17. Carey CM, Jena AB, Barnett ML. Patterns of potential opioid misuse and subsequent adverse outcomes in Medicare, 2008 to 2012. Ann Intern Med. 2018;168(12):837-45. 
Risk Factors and Outcomes of Opioid Users with and Without Concurrent

Benzodiazepine Use in the North Carolina Medicaid Population

\section{APPENDIX A Outcomes and How They Were Measured}

\begin{tabular}{l|l}
\hline Outcome & \multicolumn{1}{c}{ Measurement Definition } \\
\hline All-cause mortality & Death date \\
\hline All-cause hospitalization & Date of inpatient encounter from an institutional claim \\
\hline All-cause outpatient ED visit & $\begin{array}{l}\text { Date of ED visit from institutional encounter with HCPCS code 9928x or professional encounter with revenue } \\
\text { code 0981 or 045x }\end{array}$ \\
\hline $\begin{array}{l}\text { Unintentional opioid overdose } \\
\text { ED=emergency department; HCPCS=Healthcare Common Procedure Coding System; ICD-10-CM=International Classification of Diseases, Tenth Revision, Clinical } \\
\text { Modification. }\end{array}$
\end{tabular}

\section{APPENDIX B ICD-10-CM Diagnosis Codes}

Diagnosis disorder (SUD) 1
Substance use

\section{ICD-10-CM}

F10.10, F10.11, F10.120, F10.121, F10.129, F10.14, F10.150, F10.151, F10.159, F10.180, F10.181, F10.182, F10.188, F10.19, F10.20, F10.21, F10.220, F10.221, F10.229, F10.230, F10.231, F10.232, F10.239, F10.24, F10.250, F10.251, F10.259, F10.26, F10.27, F10.280, F10.281, F10.282, F10.288, F10.29, F10.920, F10.921, F10.929, F10.94, F10.950, F10.951, F10.959, F10.96, F10.97, F10.980, F10.981, F10.982, F10.988, F10.99, F11.10, F11.11, F11.120, F11.121, F11.122, F11.129, F11.14, F11.150, F11.151, F11.159, F11.181, F11.182, F11.188, F11.19, F11.20, F11.21, F11.220, F11.221, F11.222, F11.229, F11.23, F11.24, F11.250, F11.251, F11.259, F11.281, F11.282, Fll.288, Fll.29, Fll.90, Fll.920, Fll.921, Fll.922, Fll.929, Fll.93, Fll.94, Fll.950, Fll.951, Fl1.959, Fl1.981, Fll.982, Fll.988, F11.99, F12.10, F12.11, F12.120, F12.121, F12.122, F12.129, F12.150, F12.151, F12.159, F12.180, F12.188, F12.19, F12.20, F12.21, F12.220, F12.221, F12.222, F12.229, F12.250, F12.251, F12.259, F12.280, F12.288, F12.29, F12.90, F12.920, F12.921, F12.922, F12.929, F12.950, F12.951, F12.959, F12.980, F12.988, F12.99, F13.10, F13.11, F13.120, F13.121, F13.129, F13.14, F13.150, F13.151 F13.159, F13.180, F13.181, F13.182, F13.188, F13.19, F13.20, F13.21, F13.220, F13.221, F13.229, F13.230, F13.231, F13.232, F13.239, F13.24, F13.250, F13.251, F13.259, F13.26, F13.27, F13.280, F13.281, F13.282, F13.288, F13.29, F13.90, F13.920, F13.921, F13.929, F13.930, F13.931, F13.932, F13.939, F13.94, F13.950, F13.951, F13.959, F13.96, F13.97, F13.980, F13.981, F13.982, F13.988, F13.99, F14.10, F14.11, F14.120, F14.121, F14.122, F14.129, F14.14, F14.150, F14.151, F14.159, F14.180, F14.181, F14.182, F14.188, F14.19, F14.20, F14.21, F14.220, F14.221, F14.222, Fl4.229, F14.23, F14.24, F14.250, F14.251, F14.259, F14.280, F14.281, F14.282, F14.288, F14.29, F14.90, F14.920, F14.921, F14.922, F14.929, F14.94, F14.950, F14.951, F14.959, F14.980, F14.981, F14.982 F14.988, F14.99, F15.10, F15.11, F15.120, F15.121, F15.122, F15.129, F15.14, F15.150, F15.151, F15.159, F15.180, F15.181, F15.182, F15.188, F15.19, F15.20, F15.21, F15.220, F15.221, F15.222, F15.229, F15.23, F15.24, F15.250, F15.251, F15.259, F15.280, F15.281, F15.282, F15.288, F15.29, F15.90, F15.920, F15.921, F15.922, F15.929, F15.93, F15.94, F15.950, F15.951, F15.959, F15.980, F15.981, F15.982, F15.988, F15.99, F16.10, F16.11, F16.120, F16.121, F16.122, F16.129, F16.14, F16.150, F16.151, F16.159, F16.180, F16.183, F16.188, F16.19, F16.20, F16.21, F16.220, Fl6.221, F16.229, F16.24, F16.250, F16.251, Fl6.259, F16.280, F16.283, F16.288, F16.29, F16.90, F16.920, F16.921, F16.929, F16.94, F16.950, F16.951, F16.959, F16.980, F16.983, F16.988, F16.99, F17.203, F17.208, F17.209, F17.213, F17.218, F17.219, F17.223, F17.228, F17.229, F17.293, F17.298, F17.299, F18.10, F18.11, F18.120, F18.121, F18.129, F18.14, F18.150, F18.151, F18.159, F18.17, F18.180, F18.188, F18.19, F18.20, F18.21, F18.220, F18.221, F18.229, F18.24, F18.250, F18.251, F18.259, F18.27, F18.280, F18.288, F18.29, F18.90, F18.920, F18.921, F18.929, F18.94, F18.950, F18.951, F18.959, F18.97, F18.980, F18.988, F18.99, F19.10, F19.11, F19.121, F19.122, F19.129, F19.14, F19.150, F19.151, F19.159, F19.16, F19.17, F19.180, F19.181, F19.182, F19.188, F19.19, F19.20, F19.21, F19.220, F19.221, F19.222, F19.229, F19.230, F19.231, F19.232, F19.239, F19.24, F19.250, F19.251, F19.259, F19.26, F19.27, F19.280, F19.281, F19.282, F19.288, F19.29, F19.920, F19.921, F19.922, F19.929, F19.930, F19.931, F19.932, F19.939, F19.94, F19.950, F19.951, F19.959, F19.96, F19.97, F19.980, F19.981, F19.982, F19.988, F19.99, F55.0, F55.1, F55.2, F55.3, F55.4, F55.8, G62.1, I42.6, K29.20, K70.0, K70.10, K70.11, K70.2, K70.30, K70.31, K70.40, K70.41, K70.9, O99.320, O99.321, O99.322, O99.323, 099.324, O99.325, T40.0X1A, T40.0X2A, T40.0X3A, T40.0X4A, T40.0X5A, T40.0X5S, T40.1X1A, T40.1X2A, T40.1X3A, T40.1X4A, T40.2X1A, T40.2X2A, T40.2X3A, T40.2X4A, T40.3X1A, T40.3X2A, T40.3X3A, T40.3X4A, T40.4X1A, T40.4X2A, T40.4X3A, T40.4X4A, T40.601A, T40.602A, T40.603A, T40.604A, T40.691A, T40.692A, T40.693A, T40.694A, T40.7X1A, T40.7X2A, T40.7X3A, T40.7X4A, T40.7X5A, T40.7X5S, T40.8X1A, T40.8X2A, T40.8X3A, T40.8X4A, T40.901A, T40.902A, T40.903A, T40.904A, T40.905A, T40.905S, T40.991A, T40.992A, T40.993A, T40.994A, T40.995A, T40.995S, T41.3X1A, T41.3X2A, T41.3X3A, T41.3X4A

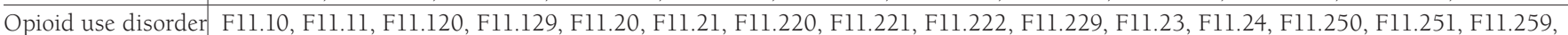
(OUD) $)^{2,3}$ F11.281, F11.282, F11.288, Fll.29, Fl1.90

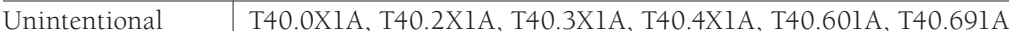

opioid overdose

Nonmetastatic and metastatic cancers ${ }^{4}$

\author{
T40.0X1A, T40.2X1A, T40.3X1A, T40.4X1A, T40.601A, T40.691A
}

C00.x-C26.x, C30.x-C34.x, C37.x-C41.x, C43.x, C45.0x-C45.2x, C45.7x, C45.9x, C46.x-C48.x, C72.x-C86.x, C88.2x-C88.4x, C88.8x,
C88.9x, C90.x-C93.x, C94.1x-C94.3x, C94.8x, C95.x, C96.0x, C96.2x, C96.4x, C96.9x, C96.Ax, C96.Zx, D03.x, D45.x, D47.Z9 in any
position 


\section{APPENDIX B ICD-10-CM Diagnosis Codes (continued)}

\begin{tabular}{|c|c|}
\hline Diagnosis & ICD-10-CM \\
\hline Chronic pain 5 & $\begin{array}{l}\text { F45.41, F45.42, G44.209, G89.0*, G89.21, G89.22, G89.28, G89.29, G89.4*, M25.50, M25.51, M25.55-M25.57, M25.78, M43.2*, } \\
\text { M43.6*, M43.8*9, M46.1*, M46.41-M46.47, M46.9*, M47.0*, M47.02, M47.1*, M47.24-M47.28, M47.814, M47.815, M47.816, M47.817, } \\
\text { M47.818, M47.894-M47.898, M48.04-M48.08, M48.1*, M48.9*, M50.0*, M50.1*, M50.3*, M50.8*, M50.9*, M51.04-M51.06, } \\
\text { M51.1*, M51.24, M51.25, M51.3*, M51.4*, M51.8*, M51.9*, M53.1*, M53.2*7, M53.2*8, M53.3*, M53.8*, M53.9*, M54.*, M60.8*, } \\
\text { M60.9*, M62.830, M67.88, M72.9*, M79.1*, M79.2*, M79.6*, M79.7*, M96.1*, M99.22-M99.29, M99.32-M99.39, M99.42-M99.49, } \\
\text { M99.52-M99.59, M99.62-M99.69, M99.72-M99.79 }\end{array}$ \\
\hline HIV6 $^{6}$ & B20, B97.35, D84.8, R75, R97.0, or R97.1 in any position \\
\hline $\begin{array}{l}\text { Schizophrenia and } \\
\text { other psychotic } \\
\text { disorders }\end{array}$ & F20.x, F21, F22, F23, F24, F25.x, F28, F29, F06.0, F06.2 in any position \\
\hline $\begin{array}{l}\text { Mood disorders } \\
\text { (i.e. bipolar, manic } \\
\text { depression, etc.) })^{7}\end{array}$ & F30.x, F31.x, F32.x, F33.x, F34.x, F39, F06.3x, R45.86 in any position \\
\hline Depression ${ }^{4}$ & $\begin{array}{l}\text { F31.3x, F31.4x, F31.5x, F31.75, F31.76, F32.x, F33.x, F34.1x, F43.10, F42.11, F43.12, F43.2x, F43.8x, F43.9x, F93.0x, F94.8x in any } \\
\text { position }\end{array}$ \\
\hline
\end{tabular}

\section{Appendix References}

1. Heslin KC, Elixhauser A. Mental and substance use disorders among hospitalized teenagers, 2012. HCUP Statistical Brief \#202. March 2016. Agency for Healthcare

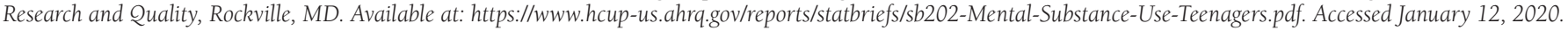

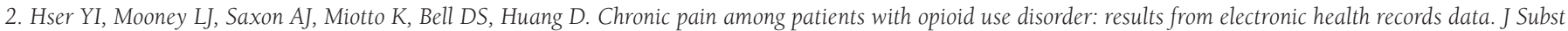
Abuse Treat. 2017;77:26-30

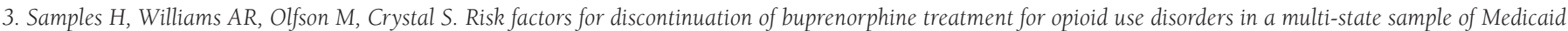
enrollees. J Subst Abuse Treat. 2018;95:9-17.

4. Quan H, Sundararajan V, Halfon P, et al. Coding algorithms for defining comorbidities in ICD-9-CM and ICD-10 administrative data. Med Care. 2005:43(11):1130-39.

5. Tian TY, Zlateva I, Anderson DR. Using electronic health records data to identify patients with chronic pain in a primary care setting. J Am Med Inform Assoc. 2013;20 (e2):e275-80.

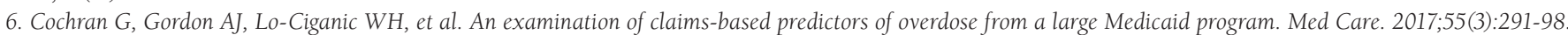

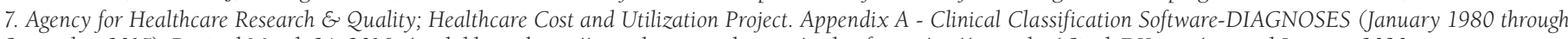
September 2015). Revised March 24, 2016. Available at: https://www.hcup-us.ahrq.gov/toolssoftware/ccs/AppendixASingleDX.txt. Accessed January 2020. 